\title{
UBL4A null mice exhibit mild kyphosis and scoliosis accompanied by dysregulation of osteoblastogenesis and chondrogenesis
}

\section{$\underline{\text { Liang } \mathrm{J}^{1}}$, $\underline{\mathrm{Li} \mathrm{J}}^{1}, \underline{\mathrm{Fu} \mathrm{Y}}^{1,2}, \underline{\operatorname{Ren}}^{1}, \underline{\mathrm{Xu} \mathrm{J}}^{3}$, $\underline{\mathrm{Zhou} \mathrm{M}}^{4}, \underline{\mathrm{Li}} \mathrm{P}^{5}$, Feng $\underline{\mathrm{H}}^{6}$, Wang $\mathrm{Y}^{1}$.}

1, State Key Laboratory of Membrane Biology, School of Medicine, Tsinghua University, Beijing, China.

2, Tsinghua University-Perking University Joint Center for Life Sciences, Beijing, China.

3, School of Biomedical Sciences, University of Western Australia, Perth, Western Australia, Australia.

4, Department of Dentistry, The First Affiliated Hospital of Guangxi Medical University, Nanning, China.

5, The General Hospital of the People's Liberation Army, Beijing, China.

6, Research Centre for Regenerative Medicine, Guangxi Medical University, Nanning, China.

\section{ABSTRACT}

Ubiquitin-like protein 4A (UBL4A) is a ubiquitin-domain protein (UDP) characterized by a ubiquitin-like domain that regulates the movement of misfolded proteins from the endoplasmic reticulum (ER) membrane to proteasome. However, its role in skeletal biology is not clear. Here we report that UBL4A plays a crucial role in skeletal development with mice lacking $U B L 4 A$ exhibiting skeletal dysplasias, mild kyphosis and scoliosis. During embryonic stage, Ubl4A knockout mice display decreased bone mineral density and trabecular bone accompanied by delayed osteogenic formation. Ubl4A knockout mice also have blended spine and small body size. At the molecular level, Ubl4A knockout mice showed perturbed expression of osteogenesis-related genes and cartilage developmental genes, indicative of altered differentiation of mesenchymal cell lineage. Transmission electron microscopy (TEM) analysis showed dilation of the endoplasmic reticulum in knockout cells of the epiphyseal chondrocytes and columnar chondrocytes. Collectively, our results uncover $U B L 4 A$ as a novel regulator in bone development and a potential candidate gene for skeletal dysplasias.

Key words: $G d X / U B L 4 A$, bone marrow stromal cells, skeletal dysplasias, bone development 


\section{INTRODUCTION}

The skeleton is a highly dynamic organ that remodels and regenerates itself in response to biomechanical stress and physiological trauma [1]. During embryogenesis and early postnatal development, these activities are uncoupled to enable rapid bone matrix production and growth in response to high metabolic demand, a process termed bone modeling [2, 3]. Despite significant progress in understanding the transcriptional cascades that control skeletogenesis [4-6], the post-transcriptional mechanisms that drive bone modeling are not well understood.

$U B L 4 A$ (also known as $G d X$ in mammals or Get5 in yeast) is a housekeeping gene located on the $\mathrm{X}$ chromosome. The human $U B L 4 A$ gene contains four exons and encodes a protein of 157 amino acids [7]. A structural analysis revealed that UBL4A is a ubiquitin-domain protein (UDP) as it contains a ubiquitin-like domain in the $\mathrm{N}$-terminus. Moreover, UBL4A has a C-terminal domain that is homologous to the C-terminal hormonogenic site of thyroglobulin [8, 9]. Although UBL4A contains a ubiquitin-like domain at its $\mathrm{N}$ terminus, it lacks ubiquitination activity [10]. The function of UBL4A in mammalian cells is similar to the function of its yeast homolog Get5 [11, 12]. Get5 forms a sorting complex with Get4 to assist Get3 in accepting newly synthesized tail-anchored (TA) proteins from the ribosome; and it interacts with the transmembrane domains of newly released TA proteins as they are delivered to the ER [13, 14]. In mammalian cells, a 'holdase' complex, consisting of Bag6 with cofactors UBL4A and Trc35, controls the movement of misfolded proteins across the endoplasmic reticulum (ER) membrane to target the cytosolic proteasome [10]. However, the role of UBL4A in skeletal biology is not known.

In this study, we identify UBL4A, a previously uncharacterized proteasomal degradation, as an essential regulator of postnatal osteoblast maturation. Loss of $U B L 4 A$ leads to impaired type I collagen synthesis and a consequent failure of bone modeling. Our results establish a critical role for UBL4A in osteoblastic bone formation and suggest that mutations in human $U B L 4 A$ may cause skeletal dysplasias and other degenerative bone disorders. 


\section{MATERIALS AND METHODS}

\section{Mice and Primary Cell Culture}

All animal studies were performed in accordance with guidelines of the Animal Care and Use Committee of Tsinghua University. The UBL4A knockout mice were generated by our lab (Wang Y, Genesis. 2012). We generated a conditional UBL4A knockout mouse in which exon 2 was flanked by two loxP sites. The KO homozygous mice were obtained by breeding $U B L 4 A^{-/+}$and male $U B L 4 A^{-/ y}$ mice. $U B L 4 A^{-/-}$mice were maintained as homozygotes on the C57BL/6 genetic background. UBL4A knockout mice were genotyped by PCR using the primers F: 5-GACCGTTGGTGTTTGCGTTG-3 and 5-GAGTGAAGCACTGCAGCATCTG-3; the wild- type allele generated a 520-bp band, whereas the $U B L 4 A$ exon 2-deleted allele generated a 300-bp band. Mice were housed under a 12-h light-dark cycle and allowed food and water ad libitum. Primary BMSCs prepared from the femurs and tibias of four-week-old knockout mice or wildtype control mice were suspended in MesenCult MSC Basal Medium (Stem Cell Technologies, CAT\#05501). The MSCs for experimentation were passaged no more than three times. The medium was refreshed every three to four days.

\section{Cell culture and qRT-PCR}

Primary BMSCs prepared from the femurs and tibias of four-week-old $U B L 4 A$ knockout mice or wild-type control mice were suspended in MesenCult MSC Basal Medium. The MSCs for experimentation were passaged no more than three times. For bone marrow stromal cells, RNA was isolated using TRIzol reagent, then reverse-transcribed by oligo (dT) and Superscript II reverse transcriptase (Invitrogen, USA). The cDNAs were amplified using SYBR green supermix with ROX (6-carboxy-X-rhodamine). $\beta$-Actin amplification was used as an internal reference for each sample. All qPCR primer sets were purchased from Invitrogen Corporation. Thermal cycling conditions were $95^{\circ} \mathrm{C}$ for 10 min followed by 40 cycles consisting of $95^{\circ} \mathrm{C}$ for $15 \mathrm{~s}$ denaturation, $60^{\circ} \mathrm{C}$ for 3 -s annealing, and $72^{\circ} \mathrm{C}$ for 30 s extension. Comparative quantitation was performed using multiple biological replicates that were analyzed in triplicate with the same untreated WT sample designated as the calibrator across different independent experimental runs. Statistical significance was 
evaluated by an unpaired t test, assuming significant association at $\mathrm{P}<0.05$ compared with control samples.

\section{Staining of the skeleton and micro-CT scanning}

The mice were dissected and fixed in $100 \%$ ethanol overnight. The tissues were stained with Alcian blue followed by Alizarin red S solution according to standard protocols (Peters, 1977). Eight-week-old UBL4A knockout and wildtype mice were analyzed using a micro-CT system ( $\mu$ CT40, Scanco Medical, Bassersdorf, Switzerland).

\section{Histology and immunohistochemical staining}

Bone tissue samples were washed and decalcified for three weeks. The tissue sections were dehydrated in a graded series of ethanol at room temperature. Samples were embedded in paraffin and sectioned at $5 \mu \mathrm{m}$. Sections were deparaffinized in xylene and rehydrated using a graded ethanol series. Sections were stained for cartilage formation with 0.5\% (wt/vol) Alcian blue (Sigma, A9186) and 0.1\% (wt/vol) nuclear fast red (Sigma, N8002) or were stained with hematoxylin-eosin (Sigma, H9627, E4009) according to standard procedures for histological analysis. Histological sections were analyzed using a light microscope (E600; Nikon).

\section{TEM}

The tissues were fixed in half-strength Karnovsky's fixative (2\% paraformaldehyde and $2.5 \%$ glutaraldehyde in $0.1 \mathrm{M}$ sodium cacodylate buffer, $\mathrm{pH}$ 7.2), and washed in the same buffer, and postfixed in $1 \%$ aqueous osmium for one hour. The samples were then dehydrated through a series of ethanol followed by propylene oxide and embedded in Eponate 12 (Ted Pella). Thin sections were cut on a microtome (Ultramicrotome; Reichert), stained with uranyl acetate and lead citrate, and examined under a microscope (CM12; Philips). Images were captured with a retractable digital camera (MultiScan; Gatan).

\section{Statistical analyses}

All data are expressed as the mean with standard deviation. The significance of the differences between UBL4A knockout and wild-type BMSCs was determined by the 
Mann-Whitney U test; $\mathrm{p}<0.05$ was considered significant.

\section{RESULTS}

\section{UBL4A-deficient mice display developmental dysplasia in bone}

Our laboratory has previously found that UBL4A can specifically stabilize the TC45/STAT3 association and promote dephosphorylation of STAT3 to repress tumorigenesis [15]. To further investigate the biological functions of UBL4A, we continued to generate UBL4A -deficient mice and found that $10 \%$ of the mice were smaller in body size compared to wild-type mice. The surviving UBL4A knockout newborns, however, appeared to be normal despite modestly lower body weight and slower growth rate (Fig. 1A, B and C). UBL4A knockout mice display severe abnormal spinal curvature (Fig. 1D). To determine if the smaller body size and abnormal spine of $U B L 4 A$ knockout mice were caused by congenital dysplasia of the bone, we performed micro-CT scanning and found that UBL4A knockout mice had typical mild scoliosis and kyphosis (Fig. 1D). These phenotypes of UBL4A knockout mice are similar to osteogenesis imperfecta in human. Thus, these defects in bone development may contribute, at least in part, to the reduced birth rate and growth retardation in $U B L 4 A$ knockout mice.

\section{Characterization of the bone dysplasia phenotype in UBL4A-deficient embryos}

Alizarin red and Alcian blue double staining of E16.5 and E18.5 mouse skeletons revealed a smaller skeleton size in $U B L 4 A$ knockout mice compared with the wild-type mice (Fig. 2A). The degree of mineralized bone in calvarias was low in E16.5 of UBL4A knockout embryos (Fig. 2B). Apparently, the degree of ossification of vertebrate was also lower than in wild-type mice (Fig. 2C). The ossification process was incomplete in the phalanx in $U B L 4 A$ knockout mice (Fig. 2D). The radius and ulna were shorter in $U B L 4 A$ knockout embryos. The gap between the radius and ulna was large in $U B L 4 A$ knockout embryos. However, the gaps between clavicle and scapula were smaller in $U B L 4 A$ knockout embryos compared with that of wild-type embryos (Fig. 2E). Von Kossa and histological staining showed that bone mineral density was decreased in UBL4A knockout mice compared with wild-type mice (Fig. 
2F). Skeletal staining with Alizarin red and Alcian blue revealed that mineralization was markedly reduced in $U B L 4 A$ knockout embryo skeletons.

The growth plate is a developmental center that integrates many signaling pathways to regulate the patterning and growth of long bones. As cells progress through the growth plate, moving from the epiphysis of the long bone towards the diaphysis, their shape and function change drastically. To distinguish whether skeletal dysplasia occurred as a consequence of an abnormal growth plate, we examined the growth plate of $U B L 4 A$ knockout mice using histological staining. It was revealed that the width of the tibial epiphysis and the length of the tibia were not significantly affected over the period of 3 to 20 weeks, although the length of the tibia was slightly reduced in UBL4A knockout mice in week 3 (Fig. 3A). Histologically, cell density was increased in the resting zone and proliferative zone of $U B L 4 A$ knockout mice, with no changes in the hypertrophic zone (Fig. 3B).

\section{Mice lacking $U B L 4 A$ have reduced postnatal bone acquisition}

To determine the importance of $U B L 4 A$ in postnatal bone development, we analyzed the skeletal phenotype of mice lacking $U B L 4 A$. X-ray images indicated that the bone density of $U B L 4 A$ knockout mice was lower than that of WT mice at 2 weeks, 4 weeks, 8weeks, and 24 weeks old (Fig. 4A). There were also changes in chondrocyte number and morphology in the articular cartilage of UBL4A knockout mice. The tidemark (between the red and yellow areas) indicated that the mineralization of articular cartilage was accelerated by deleted expression of UBL4A. In addition, a decrease in both cortical bone thickness and the length of trabecular bone was observed (distance between two arrowheads).

There was obvious trabecular bone loss in both the secondary ossification center and the metaphysis area (Fig. 4B). The loss of $U B L 4 A$ in osteoblasts dramatically impaired postnatal trabecular bone acquisition (Fig. 4B-E). Whereas the control mice accumulated trabecular bone to maximal levels by eight weeks (Fig. 4D). The mice lacking $U B L 4 A$ failed to accumulate trabecular bone; trabecular bone volume (BV/TV) was decreased by more than $35 \%$ in $U B L 4 A$ knockout mice compared to controls at both two and four weeks (Fig. 4B,C). This defect in bone acquisition was characterized by significant decreases in trabecular number (Tb. N) and thickness 
(Tb.Th.) and a significant increase in trabecular spacing (Tb. Sp). Static and dynamic histomorphometric analyses performed at two and four weeks showed that the decrease in bone mineral density in $U B L 4 A$ knockout mice was secondary to a reduction in the trabecular thickness. The differences between these two groups were more obvious in 4-week-old bones (Fig. 4C).

\section{Differentiation is perturbed in UBL4A knockout osteoblasts}

Alizarin red staining showed that the osteogenic differentiation of bone marrow stromal cells (BMSCs) was delayed after UBL4A was deleted, as shown by decreased mineralization (Alizarin red S), but the $U B L 4 A$ knockout cells had increased accumulation of sulfated glycosaminoglycans (Alcian blue) (Fig. 5A). The expression of RUNX2 measured by IHC is decreased in tibia of UBL4A knockout mice (Fig. 5B).

RNA was isolated from the femurs and tibias of $U B L 4 A$ knockout and wild-type mice. Real-time PCR experiments showed that up-regulated expression of chondrocyte cell marker genes including sox9, acan and col2a in UBL4A knockout mice (Fig. 5C), and altered ostegenic genes expression with down regulated expression of RunX2, OSX, BPS, and up regulated expression of col1a and OCN (Fig. $5 \mathrm{D})$. These data demonstrate that loss of $U B L 4 A$ leads to the dysregulation of osteoblast and chondrocyte differentiation from MSCs, suggesting that UBL4A activity is required for the normal progression of osteoblast and chondrocyte differentiation from MSCs.

\section{Perturbed endoplasmic reticulum organization in UBL4A knockout chondrocytes}

To further investigate how deletion of $U B L 4 A$ affected the skeletal system at subcellular levels, we performed a transmission electron microscopy (TEM) analysis of the developing chondrocytes in E17.5 growth plates (Fig. 6). Notably, there was dilation of the endoplasmic reticulum in knockout cells than in WT cells of the epiphyseal chondrocytes and columnar chondrocytes. In addition to the presence of intracytoplasmic fibers, the cytoplasm was filled with dilated, irregular rough endoplasmic reticulum that was engorged with abnormal, poorly formed extracellular 
matrix (ECM) in UBL4A knockout chondrocytes. 


\section{DISCUSSION}

The most conspicuous feature of the UBL4A-deficient phenotype is its acute onset, progressing from a developmentally delayed but intact skeleton at birth to severely impaired bone formation. Coincident with skeletal abnormality, we uncovered an abrupt failure of altered differentiation of osteoblast and chondrocyte mesenchymal cell lineage from MSCs in $U B L 4 A$ knockout mice.

Our results identify $U b l 4 A$ as a new gene that can regulate osteoblast activity and promote bone formation. This conclusion is supported by the following evidence. First, UBL4A knockout mice showed a series of skeletal dysplasia phenotypes, which were similar to osteogenesis imperfecta in human. Secondly, bone development abnormalities have emerged from the embryonic stage of UBL4A knockout mice. The expression of osteoblast-related genes and cartilage genes was altered in $U B L 4 A \mathrm{KO}$ long bone tissues. Thirdly, endoplasmic reticulum was extended and extracellular matrix fibers were decreased in the growth plates of $U B L 4 A$ knockout mice.

It is implicated that $U B L 4 A$ plays important roles in a broad range of BAG6 complex disorders [12]. The BAG6 complex facilitates the ER-associated degradation (ERAD) of certain aberrant membrane proteins; and it plays an important role in the ubiquitination and degradation of mislocalized membrane and secretory proteins that fail to reach the ER and remain in the cytosol [16]. It is possible that UBL4A and BAG6 may underlie a general mechanism that is required for the ubiquitin-dependent degradation of aberrant nascent chains upon their release from the ribosome, especially in the osteoblast and chondrocyte differentiation process.

Noticeably, UBL4A null mice exhibit mild kyphosis and scoliosis in adult. This would be in part due to the skeletal dysplasia and osteogenesis imperfecta UBL4A null mice. However, it remains to be determined if UBL4A plays an independent role in the development of scoliosis in mice, and is associated with human scoliosis. 


\section{Acknowledgements}

This work was supported in part by Western Australia Medical \& Health Research

Infrastructure Fund, Arthritis Australia foundation, The University of Western Australia (UWA) Research Collaboration Awards, and the Australian Health and Medical Research Council (NHMRC, No 1107828, 1027932).

\section{Conflict of interests}

The authors declare no competing or financial interests. 


\section{References}

1. Sohaskey ML, Jiang Y, Zhao JJ, Mohr A, Roemer F, Harland RM: Osteopotentia regulates osteoblast maturation, bone formation, and skeletal integrity in mice. $J$ Cell Biol, 189(3):511-525.

2. Eagle MJ, Rooney P, Kearney JN: Production of an osteoinductive demineralised bone matrix powder without the use of organic solvents. Cell Tissue Bank, 16(3):433-441.

3. Rosen CJ: Bone remodeling, energy metabolism, and the molecular clock. Cell Metab 2008, 7(1):7-10.

4. Rolfe R, Roddy K, Murphy P: Mechanical regulation of skeletal development. Curr Osteoporos Rep, 11(2):107-116.

5. Ettensohn CA: Encoding anatomy: developmental gene regulatory networks and morphogenesis. Genesis, 51(6):383-409.

6. Steinberg J, Zeggini E: Functional genomics in osteoarthritis: Past, present and future. $J$ Orthop Res.

7. Yang F, Skaletsky H, Wang PJ: Ubl4b, an X-derived retrogene, is specifically expressed in post-meiotic germ cells in mammals. Gene Expr Patterns 2007, 7(1-2):131-136.

8. Chartron JW, VanderVelde DG, Clemons WM, Jr.: Structures of the Sgt2/SGTA dimerization domain with the Get5/UBL4A UBL domain reveal an interaction that forms a conserved dynamic interface. Cell Rep, 2(6):1620-1632.

9. Wang Q, Liu Y, Soetandyo N, Baek K, Hegde R, Ye Y: A ubiquitin ligase-associated chaperone holdase maintains polypeptides in soluble states for proteasome degradation. Mol Cell, 42(6):758-770.

10. $\mathrm{Xu} \mathrm{Y,} \mathrm{Cai} \mathrm{M}$, Yang $\mathrm{Y}$, Huang L, Ye Y: SGTA recognizes a noncanonical ubiquitin-like domain in the Bag6-Ubl4A-Trc35 complex to promote endoplasmic reticulum-associated degradation. Cell Rep, 2(6):1633-1644.

11. Wu Q, Tang SG, Yuan ZM: Gremlin 2 inhibits adipocyte differentiation through activation of Wnt/beta-catenin signaling. Mol Med Rep 2015, 12(4):5891-5896.

12. Kuwabara N, Minami R, Yokota N, Matsumoto H, Senda T, Kawahara H, Kato R: Structure of a BAG6 (Bcl-2-associated athanogene 6)-Ubl4a (ubiquitin-like protein 4a) complex reveals a novel binding interface that functions in tail-anchored protein biogenesis. $J$ Biol Chem, 290(15):9387-9398.

13. Gristick HB, Rao M, Chartron JW, Rome ME, Shan SO, Clemons WM, Jr.: Crystal structure of ATP-bound Get3-Get4-Get5 complex reveals regulation of Get3 by Get4. Nat Struct Mol Biol, 21(5):437-442.

14. Tung JY, Li YC, Lin TW, Hsiao CD: Structure of the Sgt2 dimerization domain complexed with the Get5 UBL domain involved in the targeting of tail-anchored membrane proteins to the endoplasmic reticulum. Acta Crystallogr D Biol Crystallogr, 69(Pt 10):2081-2090.

15. Wang Y, Ning H, Ren F, Zhang Y, Rong Y, Wang Y, Su F, Cai C, Jin Z, Li Z et al: GdX/UBL4A specifically stabilizes the TC45/STAT3 association and promotes dephosphorylation of STAT3 to repress tumorigenesis. Mol Cell, 53(5):752-765.

16. $\mathrm{Xu} \mathrm{Y,} \mathrm{Liu} \mathrm{Y,} \mathrm{Lee} \mathrm{JG,} \mathrm{Ye} \mathrm{Y:} \mathrm{A} \mathrm{ubiquitin-like} \mathrm{domain} \mathrm{recruits} \mathrm{an} \mathrm{oligomeric} \mathrm{chaperone} \mathrm{to} \mathrm{a}$ retrotranslocation complex in endoplasmic reticulum-associated degradation. $J$ Biol Chem, 288(25):18068-18076. 


\section{Figure legends}

Fig 1. UBL4A knockout mice display multiple striking skeletal abnormalities.

(A) The body size of UBL4A knockout mice is smaller than WT mice from 1 day to 3 weeks after birth. (B) The growth of $U B L 4 A$ knockout mice is significantly retarded compared with wild-type mice ( $<<0.001$ by one-way ANOVA). (C) The body weight of wild-type mice and $U B L 4 A$ knockout mice was measured from four weeks to sixteen weeks postnatally. (D) UBL4A knockout mice show excessive spinal bending under Micro-CT.

Fig 2. Impaired skeletal ossification in the $U B L 4 A$ knockout embryos and adults mice. (A)Alizarin red and Alcian blue double staining of E16.5 and E18.5 skeletons. (B) Calvaria, (C) vertebra, (D) phalanx, (E) radial and ulna of E18.5 embryos. (F) Von Kossa staining of tibial in E17.5 WT and UBL4A knockout mice.

Fig 3. Chondrogenesis and bone development of wild-type and UBL4A knockout littermates.

(A) Quantitative analysis of the width of the tibial epiphysis (mm). (B) Quantitative analysis of the length of the tibia (mm). (C) Growth plate analysis of wild-type and UBL4A knockout mice by hematoxylin/eosin and alcian blue staining.

Fig 4. X-ray and Micro-CT scanning analysis of femurs in WT and UBL4A knockout mice.

A. Comparison of long bone between wild-type and UBL4A knockout mice by X-rays. In both 2-week-old and 24-week-old mice, the bone density of UBL4A knockout mice was lower than wild-type mice; and the cortical bone of wild-type mice (yellow arrows) was wider than that of UBL4A knockout mice (red arrows). The trabecular bone under the growth plate in UBL4A knockout mice (between red arrowheads) was shorter than in WT mice (between yellow arrowheads).

(B-E) Three-dimensional microstructural analysis of long bone and trabecular bone under the growth plate from 2 weeks to 24 weeks old mice by Micro-CT .

Osteoblast parameters were analyzed for both wild-type and UBL4A knockout mice. These parameters include bone volume divided by total volume, bone surface divided by bone volume (BS/BV), trabecular thickness (Tb.Th), trabecular number (TbN), 
trabecular spacing and bone mineral density (Tb.Sp).

Fig 5. Changes in the differentiation capacity of BMSCs isolated from the long bones of both wild-type and $U B L 4 A$ mice.

(A) Representative images of primary BMSCs by Alcian blue staining and Alizarin red staining after culturing in osteogenic or chondrogenic differentiation medium for 14 days. (B) IHC stained of tibias from wild-type and UBL4A knockout by RUNX2. (C) RT-PCR quantitation of chondrogenic differentiation-related genes from the long bone of wild-type and $U B L 4 A$ knockout mice. (D) RT-QPCR quantitation of osteogenic differentiation-related genes from the long bone of wild-type and $U B L 4 A$ knockout mice.

Fig 6. Comparative TEM images of the epiphyseal chondrocytes and columnar chondrocytes from wild-type and UBL4A knockout mice.

The endoplasmic reticulum within chondrocytes is grossly distended (A,B and C). The collagen fibrils in the extracellular matrix (ECM) are reduced in the proliferative zone of the growth plate in UBL4A knockout mice (D). ER distention is evident in the majority of UBL4A knockout cells, along with a concomitant reduction in extracellular collagen fibril assembly. 

A

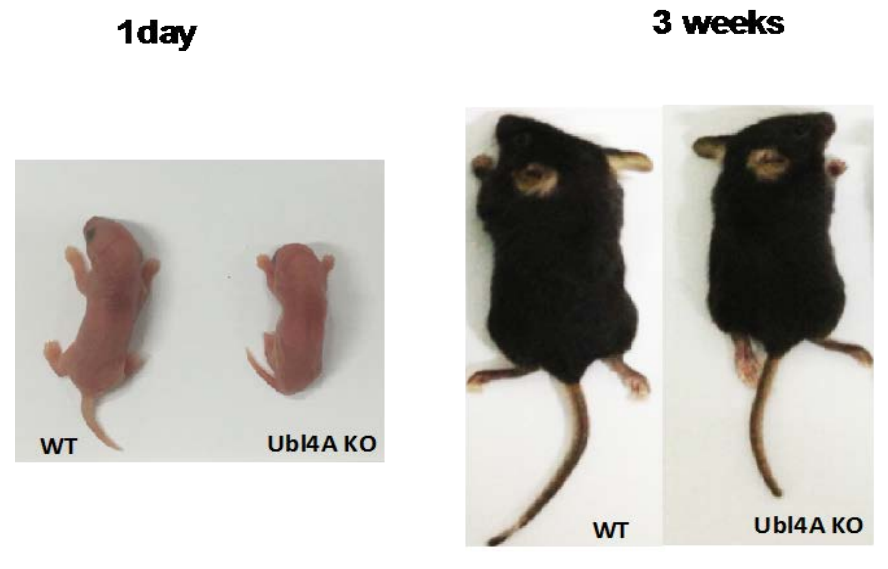

B

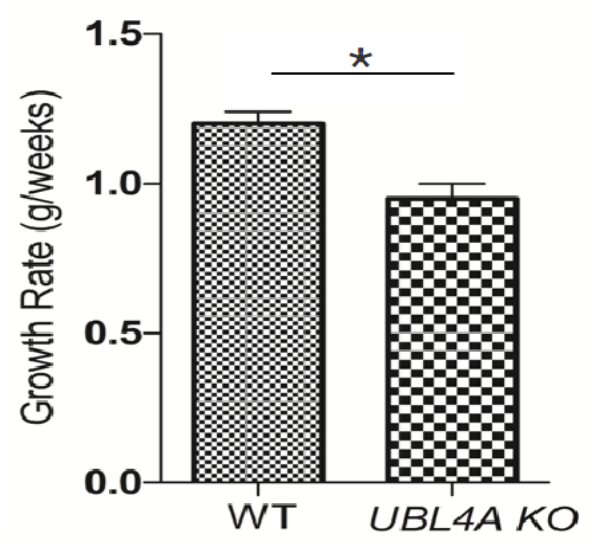

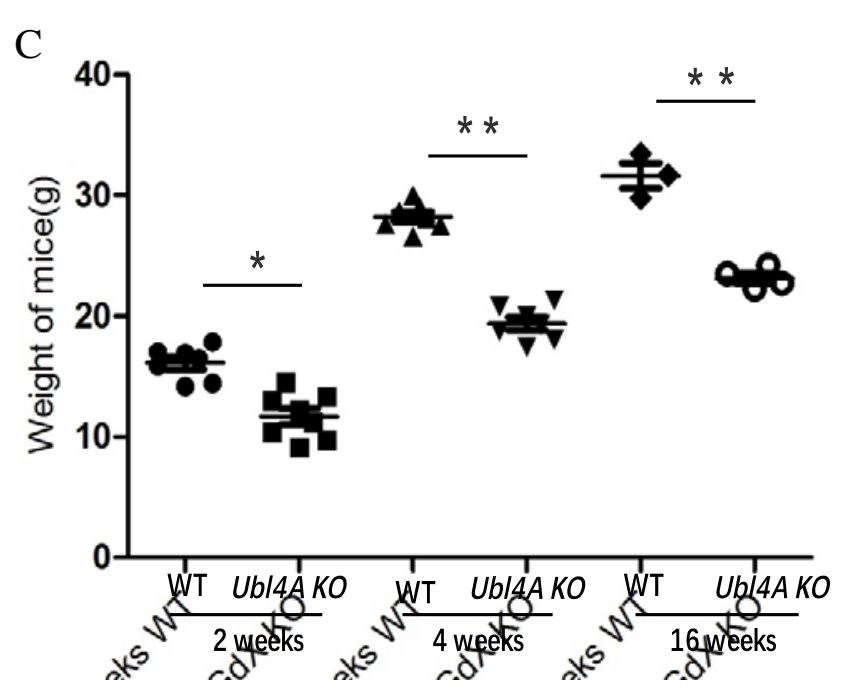

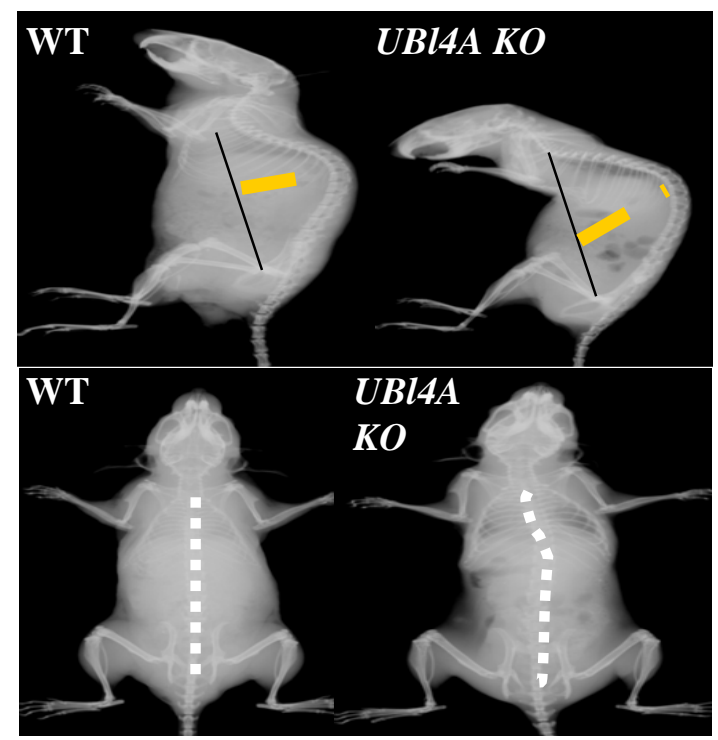

Fig 1. UBL4A knockout mice display multiple skeletal abnormalities. 
A

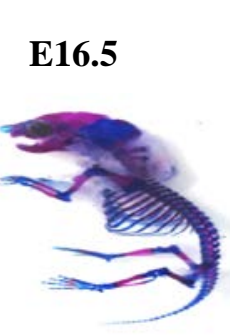

E18.5

B

Calvaria

UBL4A KO
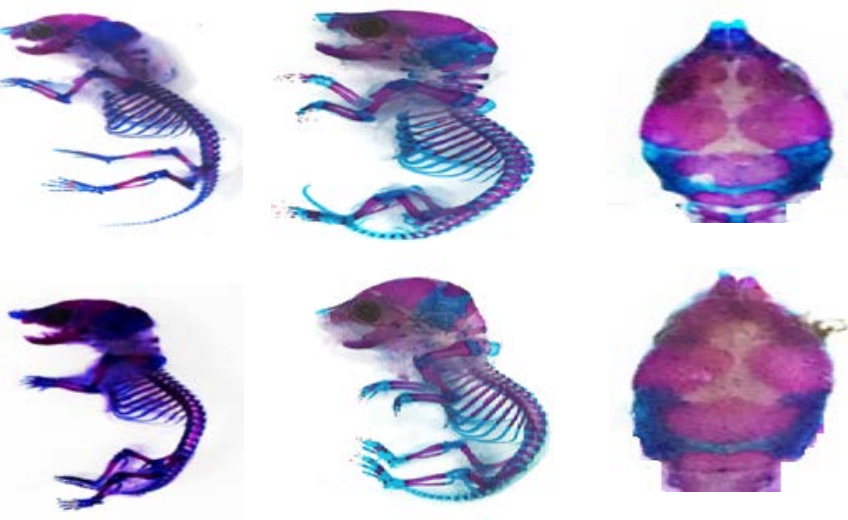

C

D

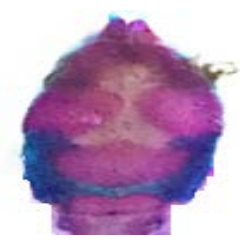

UBL4A KO

Vertebrat

Phalan

E

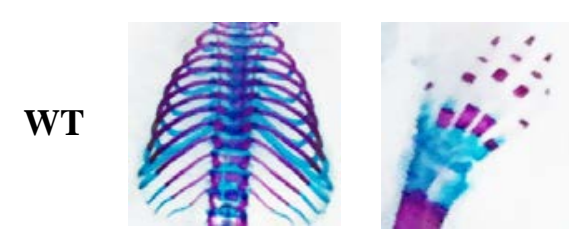

Radius \& Ulna

$\mathrm{F}$
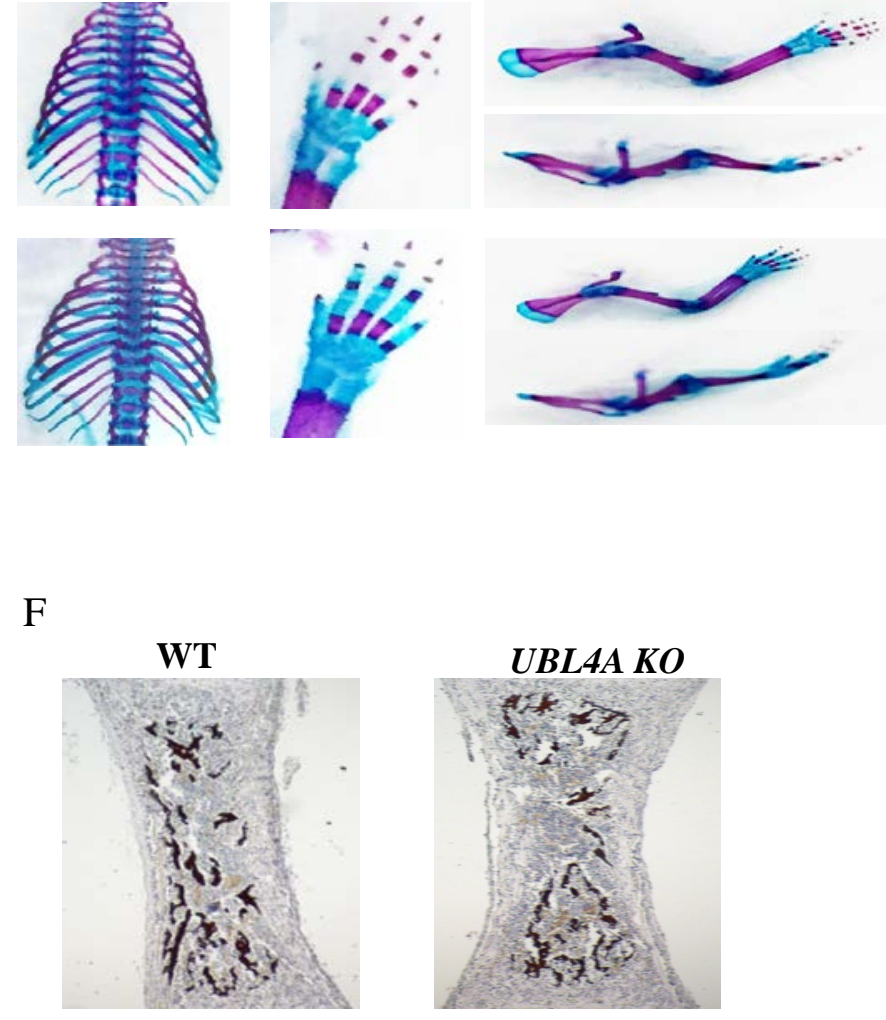

Fig 2. Impaired skeletal ossification in the UBL4A knockout embryos and mice. 
A

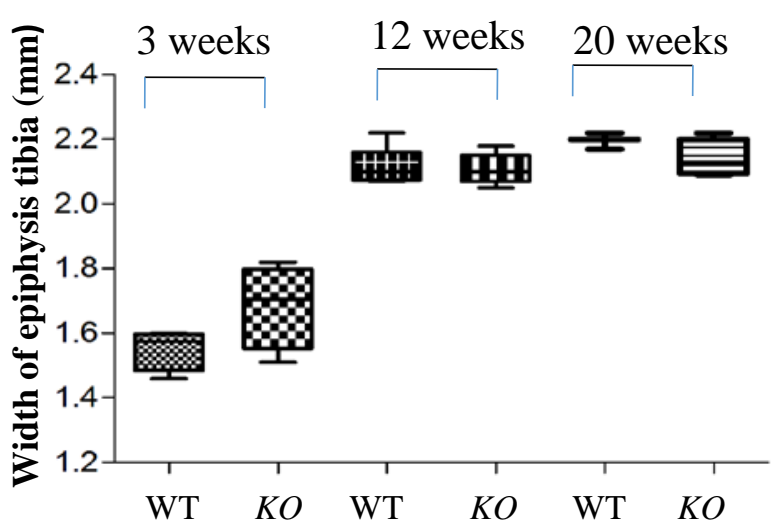

B

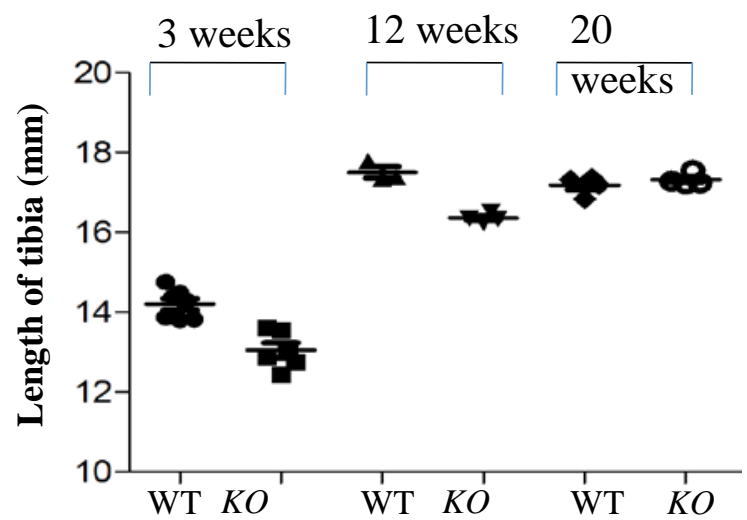

C

Resting zone Proliferative zone Hypertrophic zone Trabecular zone

UBL4A KO
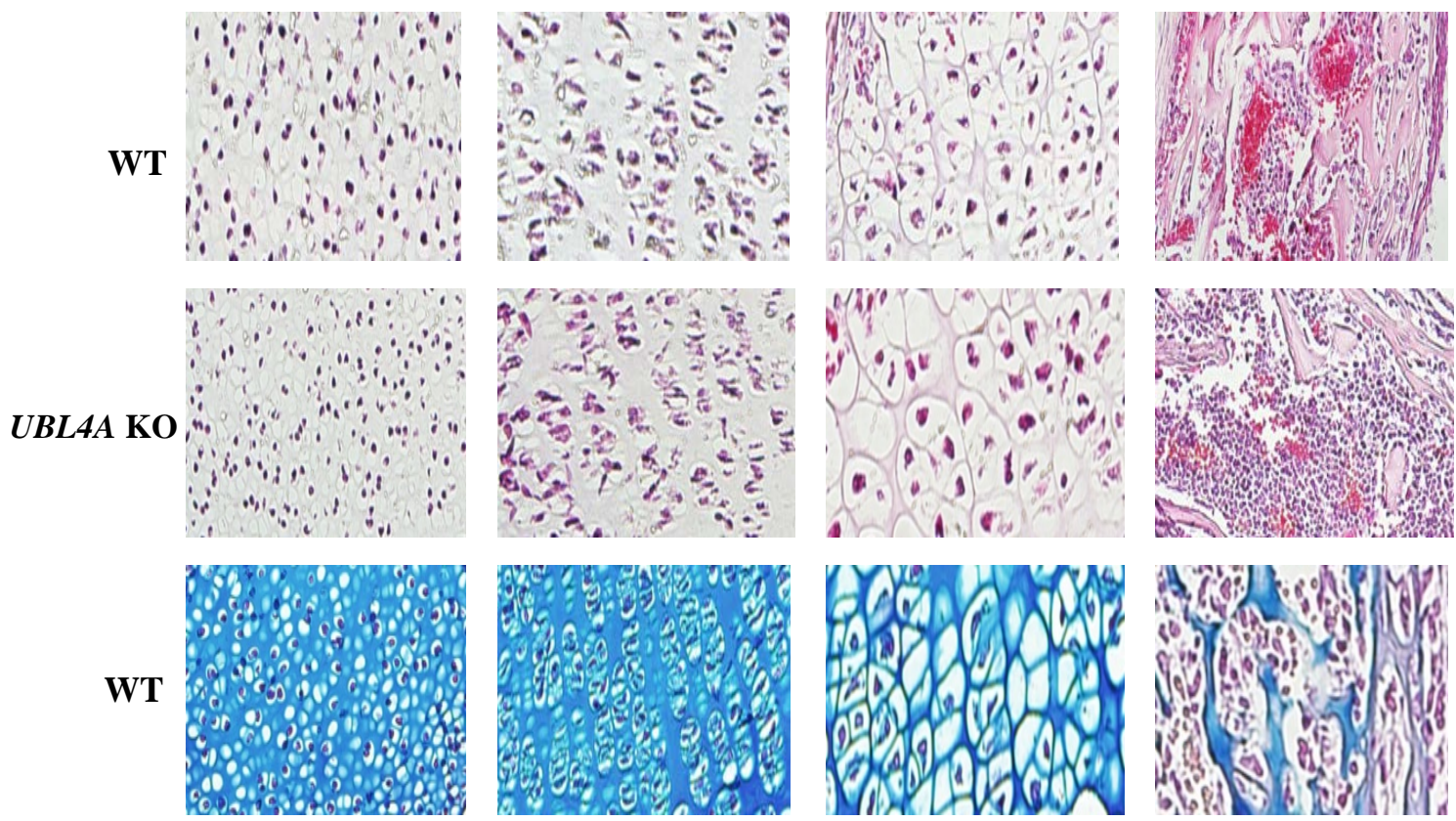

UBL4A KO
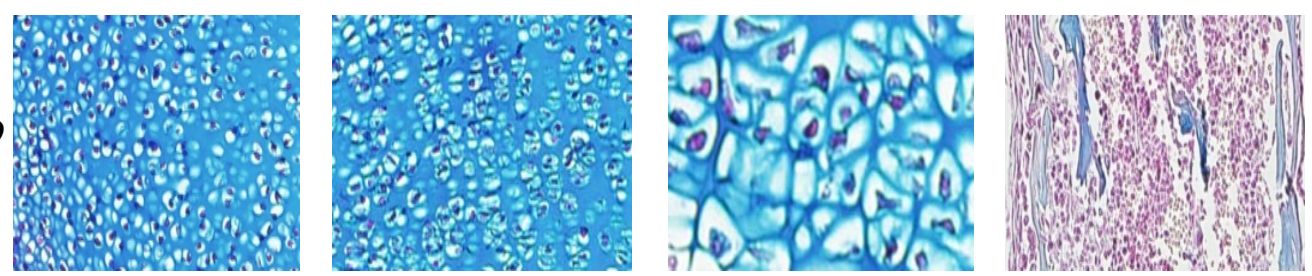

Fig3. Altered chondrogenesis and osteogenesis of wild-type and $U B L 4 A$ knockout littermates. 
A

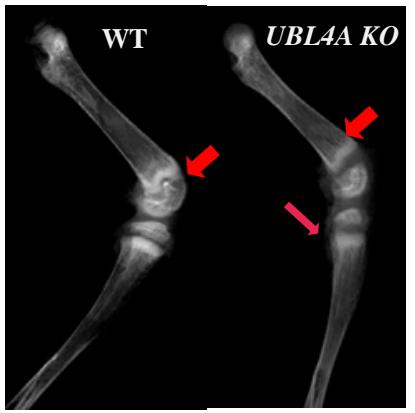

2 weeks

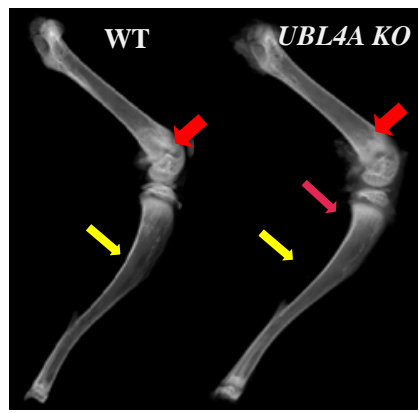

4 weeks

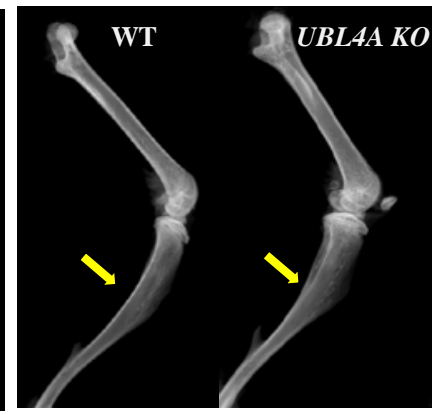

8 weeks

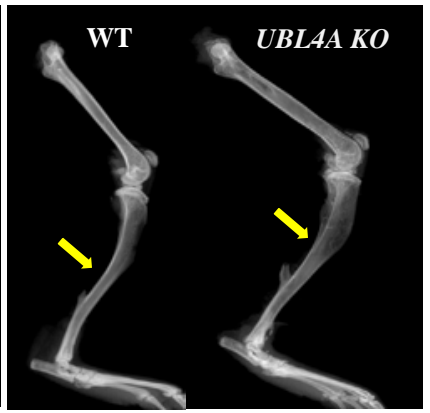

24 weeks

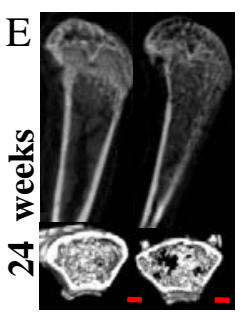

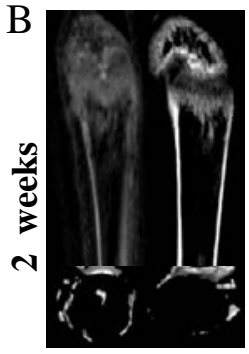
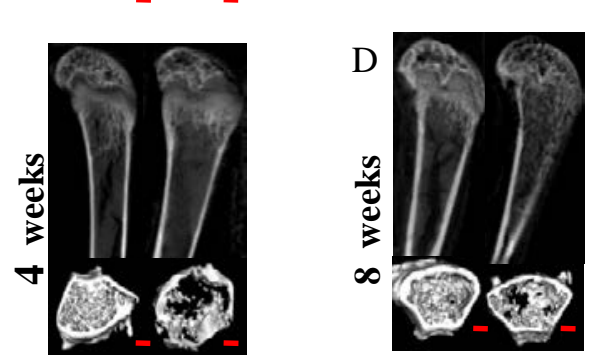

Bigger

Fig 4. X-ray and Micro-CT analysis of femurs in UBL4A KO and WT mice. 
WT $\quad \mathbf{m}$ UBLAAKO
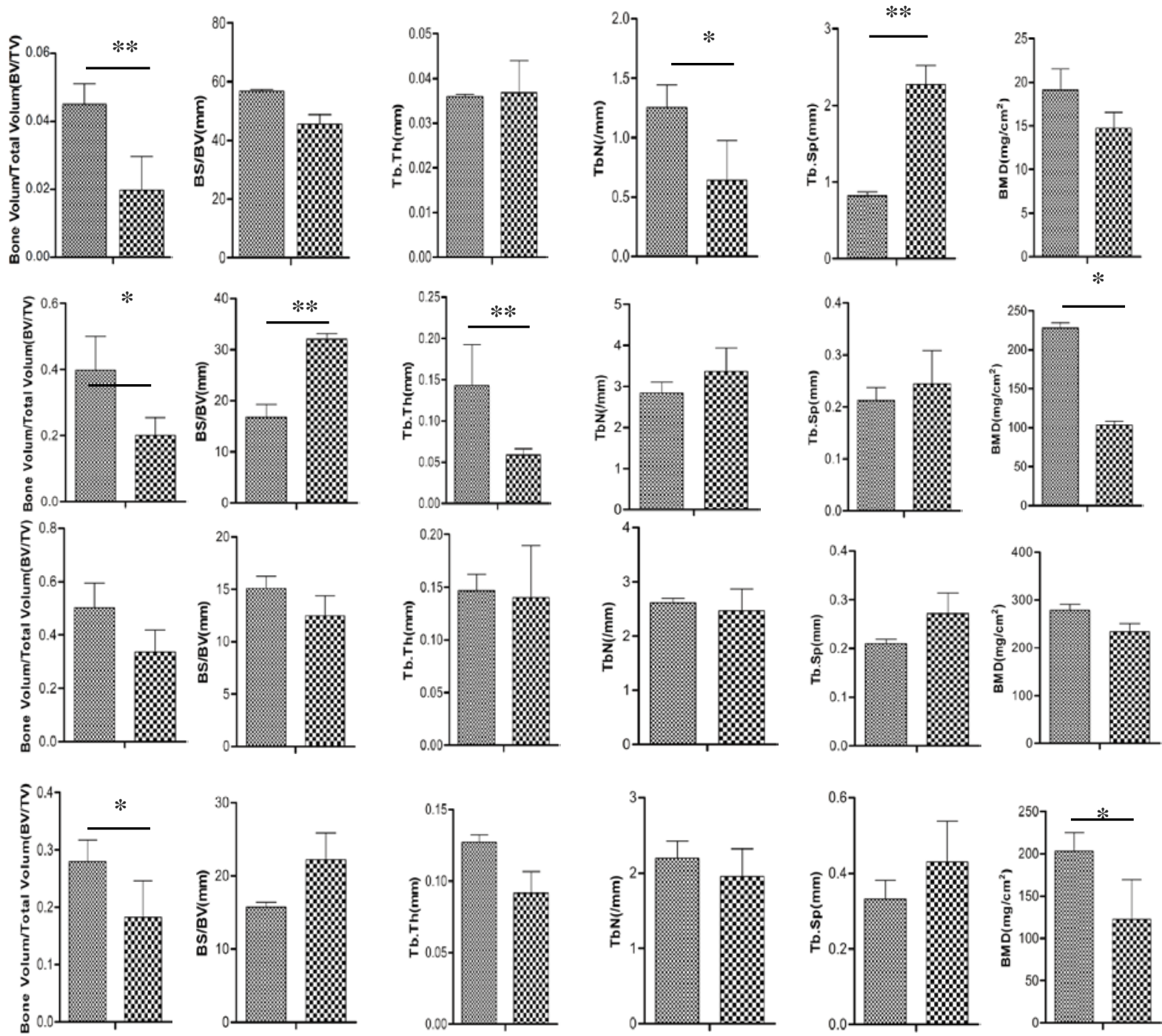

\section{Bigger, font bigger, full black and white}


A

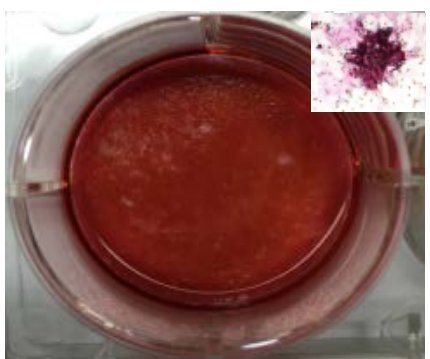

WT

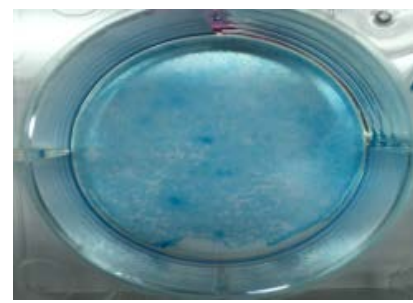

WT
B

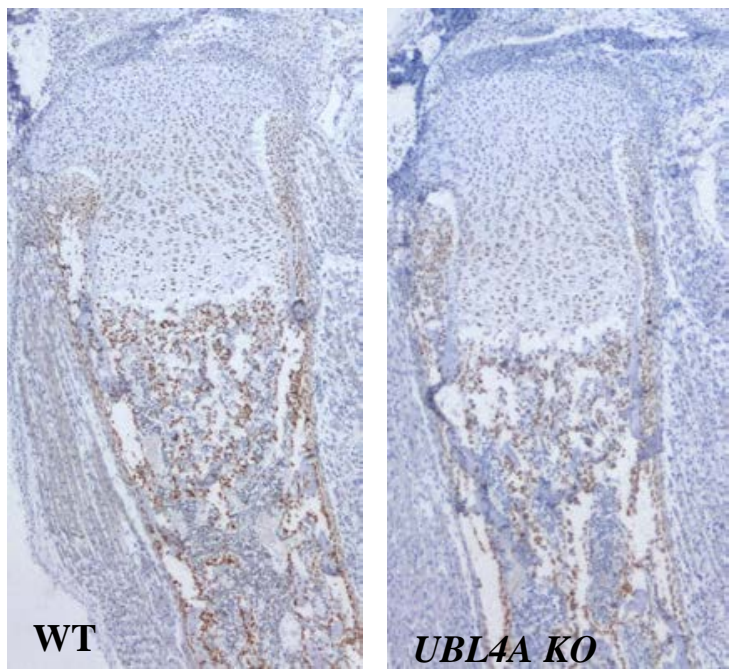

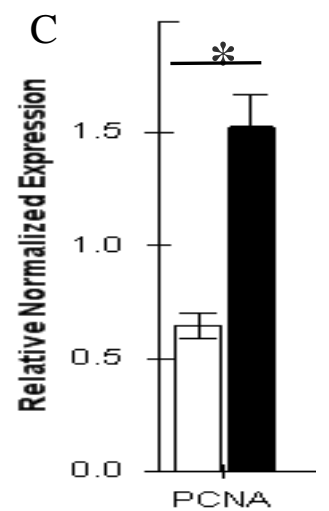
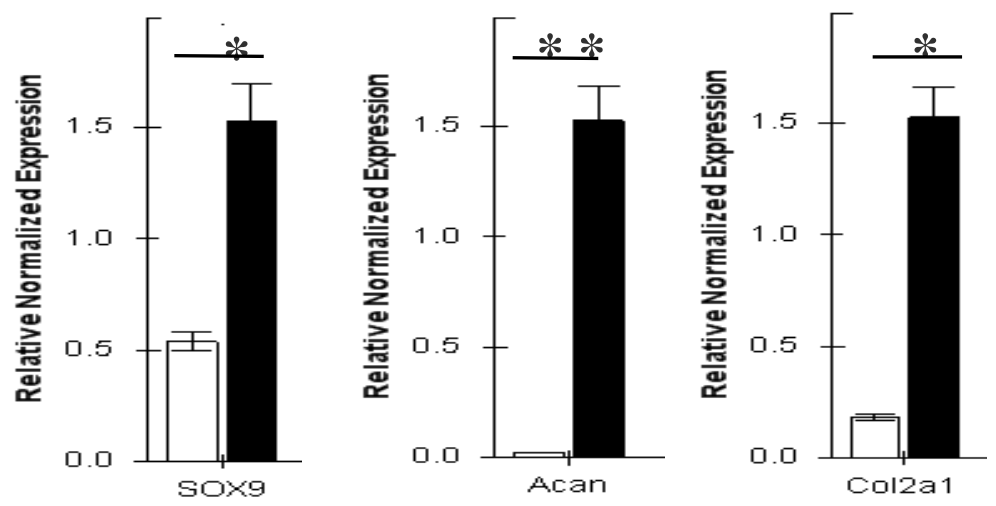

$\square_{\text {WT }}$

UBL4A KO

D
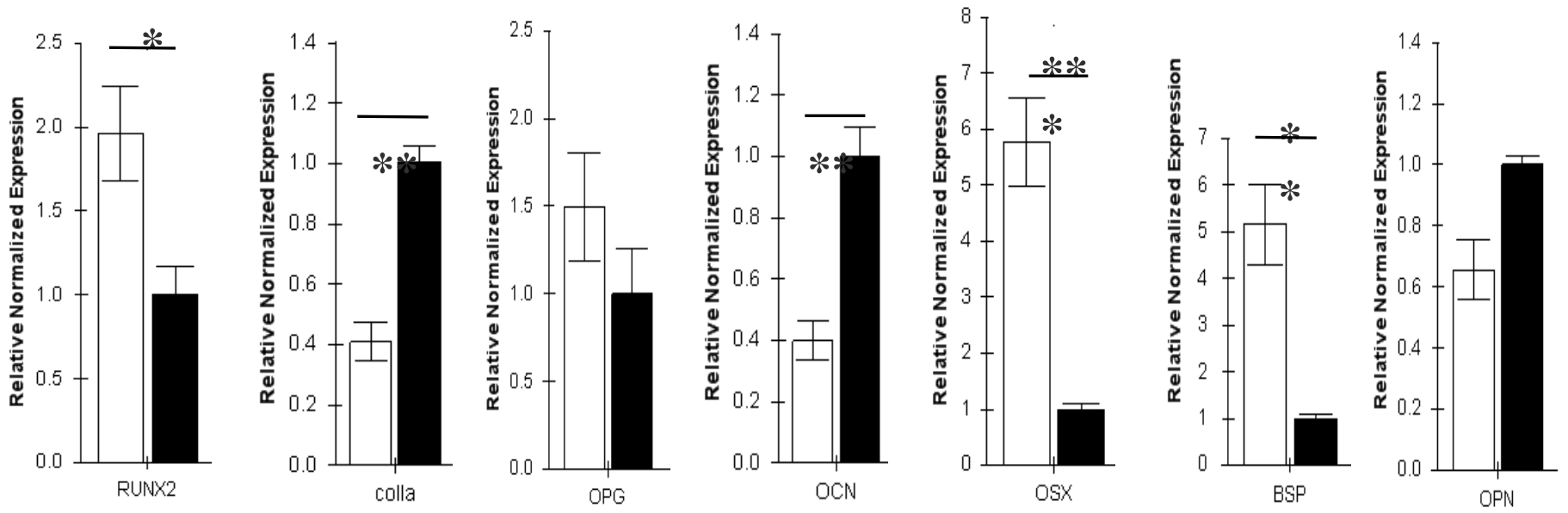

Fig 5. Changes in the differentiation capacity of BMSCs isolated from the long bones of both wild-type and $U B L 4 A$ mice. 

A
B
C
D

Epiphyseal Chondrocyte rough ER
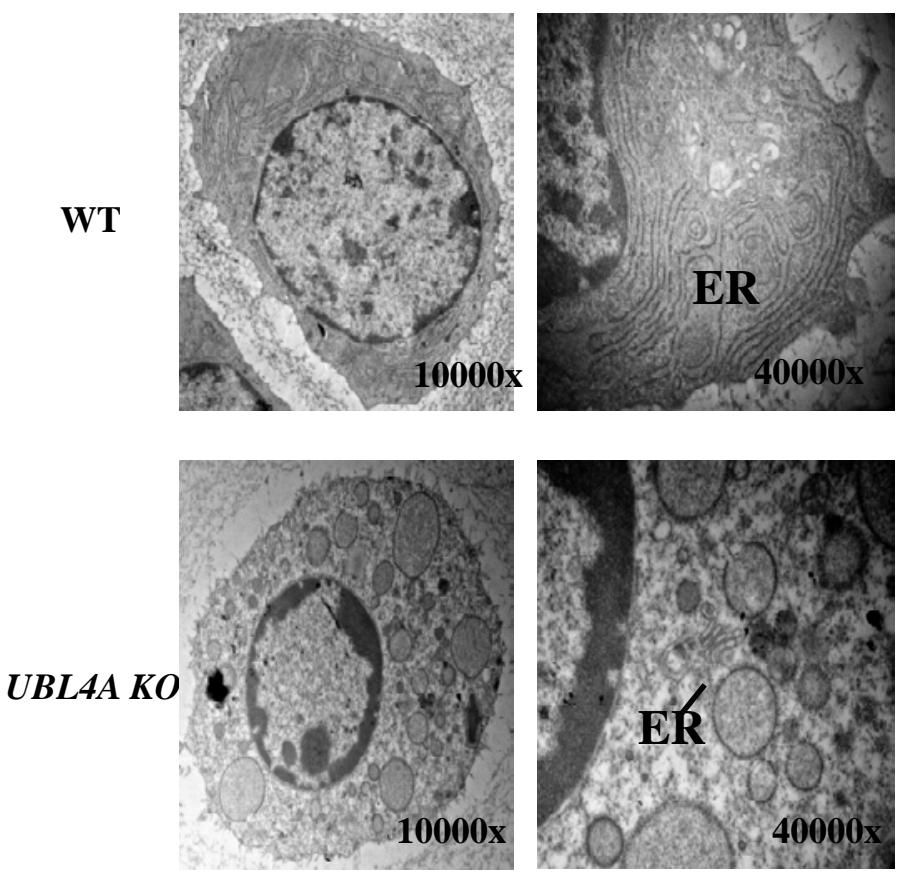

Low Magn.

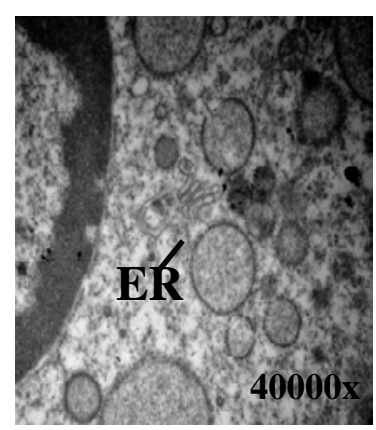

High Magn.
Columnar Chondrocyte
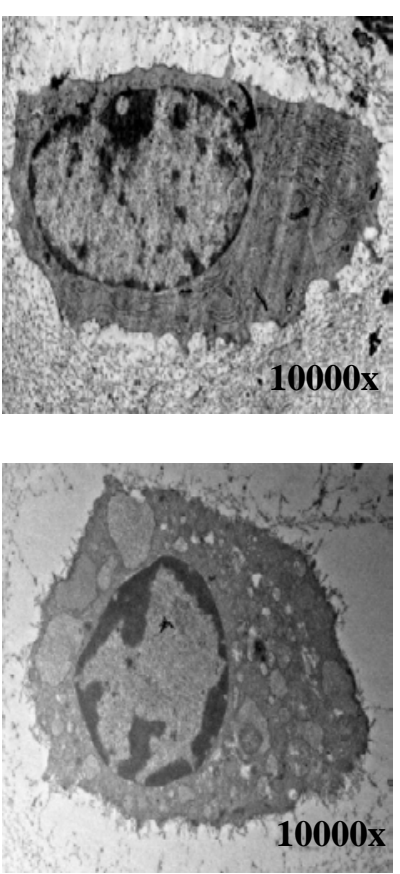

Low Magn.

\section{ECM}
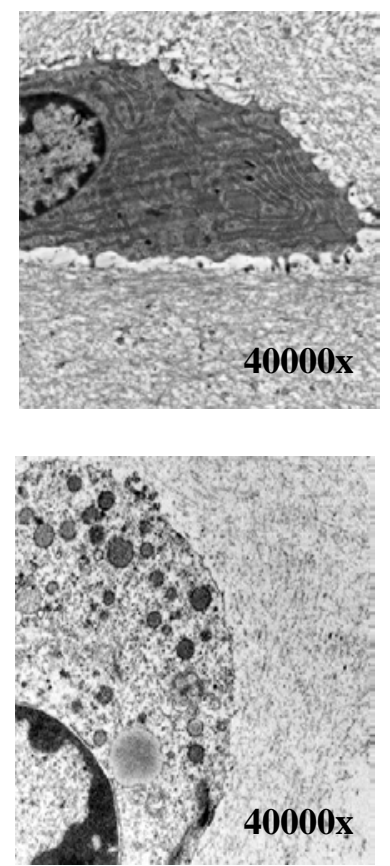

High Magn.

Fig 6. Comparative TEM images of the epiphyseal chondrocytes and columnar chondrocytes from wild-type and UBL4A knockout mice. 